\title{
Obliquely propagating electron acoustic solitons in magnetized plasmas with nonextensive electrons
}

\author{
H. R. Pakzad ${ }^{1}$ and K. Javidan ${ }^{2}$ \\ ${ }^{1}$ Department of Physics, Bojnourd Branch, Islamic Azad University, Bojnourd, Iran \\ ${ }^{2}$ Department of Physics, Ferdowsi University of Mashhad, 91775-1436 Mashhad, Iran \\ Correspondence to: H. R. Pakzad (pakzad@bojnourdiau.ac.ir) and K. Javidan (javidan@um.ac.ir) \\ Received: 2 September 2012 - Revised: 13 February 2013 - Accepted: 15 March 2013 - Published: 26 April 2013
}

\begin{abstract}
The problem of small amplitude electron-acoustic solitary waves (EASWs) is discussed using the reductive perturbation theory in magnetized plasmas consisting of cold electrons, hot electrons obeying nonextensive distribution and stationary ions. The presented investigation shows that the presence of nonextensive distributed hot electrons (due to the effects of long-range interactions) causes a reduction in the soliton amplitude while its width increases. The effects of the population ratio of hot to cold electrons and also the effects of the presence of magnetic field in this situation are also discussed.
\end{abstract}

\section{Introduction}

The idea of electron-acoustic (EA) mode was conceived by Fried and Gould (1961) during numerical solutions of the linear electrostatic Vlasov dispersion equation in unmagnetized, homogenous plasmas. Gary and Tokar (1985) performed a parameter survey and found conditions for the existence of the EA waves. Electron-acoustic waves (EAWs) can exist in a two temperature (cold and hot) electron plasmas (Watanabe and Taniuti, 1977) in which the cold electrons provide the inertia and the restoring force comes from the pressure of the hot electrons. The ions play the role of a neutralizing background only. These are electrostatic waves of high-frequency (in comparison with the ion plasma frequency), propagating at a phase speed which lies between the hot and cool electron thermal velocities. EAWs have been observed in laboratory experiments (Derfler and Simonen, 1969; Henry and Treguier, 1972; Ikezawa and Nakamura, 1981) and space plasmas, e.g. in Earth's bow shock (Thomsen et al., 1983; Feldman et al., 1983; Bale et al., 1998) and in the auroral magnetosphere (Tokar and Gary, 1984; Lin et al., 1984). They are associated with broadband electrostatic noise (BEN), a common high-frequency background activity, regularly observed by satellite missions in the plasma sheet boundary layer (PSBL) (Matsumoto et al., 1994; Cattell et al., 1999; Kakad et al., 2009). BEN emission includes a series of isolated bipolar pulses, within a frequency range from about $10 \mathrm{~Hz}$ up to the local electron plasma frequency (about $10 \mathrm{kHz}$ ) (Matsumoto et al., 1994). This clearly suggests that BEN is related to electron dynamics rather than to the ions (Matsumoto et al., 1994; Kakad et al., 2009). During the past decade, there has been a vast body of theoretical literature on electron-acoustic waves to explain the space observations of solitary waves with either negative (Tagare et al., 2004) or positive potentials (Mamuna et al., 2002a, b). Verheest et al. (2005) demonstrated that the inclusion of the hot electron inertia can lead to compressive electron-acoustic solitons and may render the analysis much more intricate (Verheest et al., 2007). There are several other papers dealing with electronacoustic waves (Yu and Shukla, 1983; Singh and Lakhina, 2001; Cattaert et al., 2005; Lakhina et al., 2008, 2009; Pottelette and Berthomier, 2009).

However, these investigations (Dubouloz et al., 1991; Mace et al., 1991; Berthomier et al., 2000) are not valid for obliquely propagating EASWs in magnetized plasma. The nonlinear propagation of the EA waves in a magnetized plasma has been considered by Dubouloz et al. (1993). They reported that the electric field spectrum produced by an EASW is not significantly modified by the presence of a magnetic field. Recently, Mace and Hellberg (2001) studied the properties of obliquely propagating EASWs in magnetized plasmas. They have shown that the existence of negative potential EASWs corresponding to a compression of the cold electron density is possible. 
On the other hand, space plasma observations indicate clearly the presence of electron populations which are far away from their thermodynamic equilibrium (Vasyliunas, 1968; Leubner, 1982; Armstrong et al., 1983; Gill et al., 2006; Anowar and Mamun, 2008; Pakzad and Tribeche, 2010; Danehkar et al., 2011). Over the last two decades, a great deal of attention has been paid to nonextensive statistical mechanics based on the deviations of Boltzmann-GibbsShannon (B-G-S) entropy measure. A suitable nonextensive generalization of the B-G-S entropy for statistical equilibrium was first recognized by Renyi (1955) and subsequently proposed by Tsallis (1988), suitably extending the standard additivity of the entropies to the nonlinear, nonextensive case where one particular parameter, the entropy index $q$, characterizes the degree of nonextensivity of the considered system ( $q=1$ corresponds to the standard, extensive, B-G-S statistics). Indeed, there are many physical systems which cannot be explained using the classical statistical description correctly. Some of them can be described by suitable framework of nonextensive statistics. As is well-known, the Maxwellian distribution in Boltzmann-Gibbs statistics is believed valid universally for the macroscopic ergodic equilibrium systems. However, for systems with long-range interactions, such as plasmas (Coulombian long-range interaction) and gravitational systems, with non-equilibrium stationary states, the Maxwellian distribution might be inadequate for complete description of the features. The parameter $q$ that underpins the generalized entropy of Tsallis is linked to the underlying dynamics of the system and measures the amount of its nonextensivity. Nonextensive systems (in statistical mechanics and thermodynamics) are systems for which the whole entropy is different from the sum of the entropies of the respective parts. In other words, the generalized entropy of the whole is greater than the sum of the entropies of the parts if $q<1$ (superextensivity), whereas the generalized entropy of the system is smaller than the sum of the entropies of the parts for $q>1$ (subextensivity). Nonextensive statistics was successfully applied to a number of astrophysical and cosmological scenarios. Those include stellar polytropes (Plastino and Plastino, 1993), the solar neutrino problem (Kaniadakis et al., 1996), peculiar velocity distributions of galaxies (Lavagno et al., 1998) and systems with long-range interactions and also fractal-like space-times. Cosmological implications were discussed in Torres et al. (1997), and plasma oscillations in a collisionless thermal plasmas (which has been recently analysed) were provided from $q$ statistics (Lima et al., 2000).

On the other hand, $\kappa$ distributions are highly favoured in any kind of space plasma modelling (Mendis and Rosenberg, 1994) where a reasonable physical background was not apparent. A comprehensive discussion of $\kappa$ distributions in view of experimentally favoured nonthermal tail formations is provided in Leubner and Schupfer (2000) where also typical values of the index $\kappa$ are quoted and referenced for different space plasma environments. In the present analysis the missing link to fundamental physics is provided within the framework of an entropy modification consistent with nonextensive statistics. The family of $\kappa$ distribution is obtained from the positive definite part $1 / 2 \leq \kappa \leq \infty$, corresponding to $-1 \leq q \leq 1$ of the general statistical formalism where in comparison the spectral index $\kappa$ is a measure of the degree of nonextensivity. Since the main theorems of the standard Maxwell-Boltzmann statistics admit profound generalizations within nonextensive statistics (Plastino, 1994; Rajagopal, 1995, 1996; Chame and Mello, 1997; Lenzi et al., 1998), a justification for the use of $\kappa$ distributions in astrophysical plasma modelling is provided from fundamental physics.

The effects of electron nonextensivity on the existence and possible realization of EASWs in unmagnetized plasmas have been studied in Pakzad (2011b). To study the effect of nonextensive hot electrons on small amplitude electronacoustic double-layers (EA-DLs), Pakzad and Tribeche (2011) considered unmagnetized electron and ion fluids. The plasma systems under investigation in other papers are different in master equations. Note that electron-acoustic solitons in plasmas consisting of cold and hot electrons are investigated in this paper.

Motivated by this situation, we consider a magnetized plasma model consisting of a cold, magnetized electron fluid, hot electrons obeying a nonextensive distribution, and stationary ions, and investigate the properties of obliquely propagating EASWs.

\section{Basic equations}

We consider homogeneous plasmas consisting of a cold electron fluid, hot electrons obeying a nonextensive distribution and stationary ions in the presence of an external magnetic field $\boldsymbol{B}_{0}=\boldsymbol{B}_{0} \hat{z}$. The nonlinear dynamics of the electronacoustic solitary waves is governed by the continuity and motion equations for cold electrons, and Poisson's equation (Mamun et al., 2002):

$$
\begin{aligned}
& \frac{\partial n_{\mathrm{c}}}{\partial t}+\nabla \cdot\left(n_{\mathrm{c}} u_{\mathrm{c}}\right)=0, \\
& \frac{\partial u_{\mathrm{c}}}{\partial t}+\left(u_{\mathrm{c}} \cdot \nabla\right) u_{\mathrm{c}}=\alpha \nabla \varphi-\omega_{\mathrm{cc}}\left(u_{\mathrm{c}} \cdot \hat{z}\right), \text { and } \\
& \nabla^{2} \varphi=\frac{1}{\alpha} n_{\mathrm{c}}+n_{\mathrm{h}}-\left(1+\frac{1}{\alpha}\right) .
\end{aligned}
$$

In the above equations, $n_{\mathrm{c}}\left(n_{\mathrm{h}}\right)$ is the cold (hot) electron number density normalized by its equilibrium values $n_{c 0}\left(n_{h 0}\right)$. $u_{\mathrm{c}}$ is the cold electron fluid velocity normalized by the phase speed of electron-acoustic $\left(C_{\mathrm{e}}=\left(k_{B} T_{\mathrm{h}} / \alpha m_{\mathrm{e}}\right)^{1 / 2}\right)$ in which $k_{B}$ is Boltzmann's constant, $e$ the electron charge, $m_{\mathrm{e}}$ electron mass and $\alpha=n_{h 0} / n_{c 0} \cdot \omega_{\mathrm{cc}}=\left(e B_{0} / m c\right) / \omega_{\mathrm{pc}}$ the cold electron cyclotron frequency normalized by the cold electron plasma frequency $\omega_{\mathrm{pc}}$, and $\varphi$ the electrostatic wave potential normalized by $k_{B} T_{\mathrm{h}} / e$. The time and space variables are 
in units of the cold electron plasma period $\omega_{\mathrm{pc}}^{-1}$ and the hot electron Debye radius $\lambda_{\mathrm{Dh}}$, respectively.

To model the effects of nonextensivity due to the longrange interactions between the electrons, we refer to the one-dimensional equilibrium $q$ distribution function given by Watanabe (1975),

$f_{\mathrm{e}}\left(v_{\mathrm{e}}\right)=C_{q}\left\{1+(1-q)\left[\frac{m_{\mathrm{e}} v_{\mathrm{e}}^{2}}{2 T_{\mathrm{e}}}-\varphi\right]\right\}^{\frac{1}{q-1}}$.

The constant of normalization is

$$
\begin{aligned}
& C_{q}=n_{\mathrm{e} 0} \frac{\Gamma\left(\frac{1}{1-q}\right)}{\Gamma\left(\frac{1}{1-q}-\frac{1}{2}\right)} \sqrt{\frac{m_{\mathrm{e}}(1-q)}{2 \pi T_{\mathrm{e}}}} \quad \text { for }-1<q<1 \\
& C_{q}=n_{\mathrm{e} 0}\left(\frac{1+q}{2}\right) \frac{\Gamma\left(\frac{1}{q-1}+\frac{1}{2}\right)}{\Gamma\left(\frac{1}{q-1}\right)} \sqrt{\frac{m_{\mathrm{e}}(q-1)}{2 \pi T_{\mathrm{e}}}} \text { for } q>1 .
\end{aligned}
$$

Here, the parameter $q$ stands for the strength of the nonextensivity and the quantity $\Gamma$ for the standard gamma function. It may be useful to note that, for $q>-1$, the $q$ distribution in Eq. (2) is unnormalizable. If the electron velocity distribution is the well-known Maxwell-Boltzmann distribution, the corresponding value of the parameter $q$ is unity,

$$
f_{\mathrm{e}}\left(v_{\mathrm{e}}\right)=\frac{n_{\mathrm{e} 0}}{\left(2 \pi T_{\mathrm{e}} / m_{\mathrm{e}}\right)^{1 / 2}} \exp \left(-\frac{v_{\mathrm{e}}^{2}-2 T_{\mathrm{e}} \varphi / m_{\mathrm{e}}}{2 T_{\mathrm{e}} / m_{\mathrm{e}}}\right) .
$$

Integrating Eq. (2) over the velocity space and noting that, for $q>1$, the distribution function in Eq. (2) exhibits a thermal cutoff on the maximum value allowed for the velocity of the particles, given by

$v_{\max }=\sqrt{\frac{2 T_{\mathrm{e}}}{m_{\mathrm{e}}}\left(\frac{1}{q-1}+\varphi\right)}$,

we get

$n_{\mathrm{e}}=n_{\mathrm{h}}=\left\{\begin{array}{llr}\int_{-\infty}^{+\infty} f_{\mathrm{e}}\left(v_{\mathrm{e}}\right) d v_{\mathrm{e}} & \text { for } & -1<\mathrm{q}<1 \\ \int_{-v_{\max }}^{+v_{\max }} f_{\mathrm{e}}\left(v_{\mathrm{e}}\right) d v_{\mathrm{e}} & \text { for } & q>1\end{array}\right.$.

Finally, the normalized hot electron density is given by Gougam and Tribeche (2011) and Amour and Tribeche (2010)

$n_{\mathrm{h}}=[1+(q-1) \varphi]^{\frac{q+1}{2(q-1)}}$.

\section{Solitary structure}

In order to study the electron-acoustic solitary waves in the plasma model under consideration, we construct a weakly nonlinear theory of the electrostatic waves with small but finite amplitude, which leads to a scaling of the independent variables through the stretched coordinates $\xi=\varepsilon^{1 / 2}\left(l_{\mathrm{x}} \mathrm{x}+l_{\mathrm{y}} \mathrm{y}+l_{\mathrm{z}} \mathrm{z}-\lambda t\right), \tau=\varepsilon^{3 / 2} t$, where $\varepsilon$ is a small dimensionless parameter measuring the weakness of the dispersion and nonlinearity. Parameters $l_{\mathrm{x}}, l_{\mathrm{y}}$ and $l_{\mathrm{z}}$ are the directional cosines of the wave vector $\boldsymbol{k}$ along the $\mathrm{x}-, \mathrm{y}-$, and $\mathrm{z}$-axes, respectively, so that $l_{\mathrm{x}}^{2}+l_{\mathrm{y}}^{2}+l_{\mathrm{z}}^{2}=1$. The $\lambda$ is unknown phase velocity which will be determined later. In the above transformation, $\lambda$ is normalized by $C_{\mathrm{e}}$.

We also expand $n_{\mathrm{c}}, u_{c x, y, z}$ and $\varphi$ in a power series of $\varepsilon$ as follows:

$$
\left[\begin{array}{l}
n_{\mathrm{c}}=1+\varepsilon n_{1 \mathrm{c}}+\varepsilon^{2} n_{2 \mathrm{c}}+\ldots \\
u_{c x}=\varepsilon^{3 / 2} u_{1 c x}+\varepsilon^{2} u_{2 \mathrm{cx}}+\ldots \\
u_{c y}=\varepsilon^{3 / 2} u_{1 \mathrm{cy}}+\varepsilon^{2} u_{2 \mathrm{cy}}+\ldots \\
u_{c z}=\varepsilon u_{1 \mathrm{cz}}+\varepsilon^{2} u_{1 \mathrm{cz}}+\ldots \\
\varphi=\varepsilon \varphi_{1}+\varepsilon^{2} \varphi_{2}+\ldots
\end{array}\right.
$$

We write equation set in Eq. (1) in various powers of $\varepsilon$. From the lowest order in $\varepsilon$ of the continuity equation, the $\mathrm{z} \mathrm{com-}$ ponent of the momentum equation and Poisson's equation, we have $n_{1 \mathrm{c}}=\frac{-\alpha l_{\mathrm{z}}^{2}}{\lambda^{2}} \varphi_{1}, u_{1 \mathrm{cz}}=\frac{-\alpha l_{\mathrm{z}}}{\lambda} \varphi_{1}$ and $\lambda=\sqrt{\frac{2}{q+1}} l_{\mathrm{z}}$. One can write the lowest order of the $\mathrm{x}$ and $\mathrm{y}$ components of the momentum equation as

$u_{1 c x}=\frac{-\alpha l_{\mathrm{y}}}{\omega_{\mathrm{cc}}} \frac{\partial \varphi_{1}}{\partial \xi}, \quad u_{1 \mathrm{cy}}=\frac{\alpha l_{\mathrm{x}}}{\omega_{\mathrm{cc}}} \frac{\partial \varphi_{1}}{\partial \xi}$,

which represent the $\mathrm{y}$ and $\mathrm{x}$ components of the cold electron drift arising due to the balance between the electric and Lorentz forces respectively. We can also obtain the next higher order $\mathrm{x}$ and $\mathrm{y}$ components of the momentum equation as

$u_{2 \mathrm{cx}}=\frac{-\alpha l_{\mathrm{x}} \lambda}{\omega_{\mathrm{cc}}^{2}} \frac{\partial^{2} \varphi_{1}}{\partial \xi^{2}}, \quad u_{2 \mathrm{cy}}=\frac{-\alpha l_{\mathrm{y}} \lambda}{\omega_{\mathrm{cc}}^{2}} \frac{\partial^{2} \varphi_{1}}{\partial \xi^{2}}$.

These are the $\mathrm{x}$ and $\mathrm{y}$ components of the cold electron polarization drift respectively. To the next higher order in $\varepsilon$, from the continuity equation, the $\mathrm{z}$ component of the momentum equation and Poisson's equation, we obtain

$$
\mid \begin{aligned}
& \frac{\partial n_{1 \mathrm{c}}}{\partial \tau}-\lambda \frac{\partial n_{2 \mathrm{c}}}{\partial \xi}+l_{\mathrm{x}} \frac{\partial u_{2 \mathrm{cx}}}{\partial \xi}+l_{\mathrm{y}} \frac{\partial u_{2 \mathrm{cy}}}{\partial \xi}+l_{\mathrm{z}} \frac{\partial u_{1 \mathrm{cz}}}{\partial \xi}=0 \\
& \frac{\partial u_{1 \mathrm{cz}}}{\partial \tau}-\lambda \frac{\partial u_{1 \mathrm{cz}}}{\partial \xi}+u_{1 \mathrm{cz}} l_{\mathrm{z}} \frac{\partial u_{1 \mathrm{cz}}}{\partial \xi}-\alpha l_{\mathrm{z}} \frac{\partial \varphi_{2}}{\partial \xi}=0 \\
& \frac{\partial^{2} \varphi_{1}}{\partial \xi^{2}}-\frac{n_{2 \mathrm{c}}}{\alpha}-\frac{q+1}{2} \varphi_{2}-\frac{(q+1)(3-q)}{2^{3}} \varphi_{1}^{2}=0
\end{aligned} .
$$

Finally, from Eqs. (10), (11), and (12), the KdV equation yields

$$
\frac{\partial \varphi_{1}}{\partial \tau}+A \varphi_{1} \frac{\partial \varphi_{1}}{\partial \xi}+\boldsymbol{B} \frac{\partial^{3} \varphi_{1}}{\partial \xi^{3}}=0,
$$

where the coefficients are

$$
\begin{aligned}
A & =\frac{-l_{\mathrm{z}}}{\sqrt{2(q+1)}}\left\{\frac{3-q}{2}+3\left(\frac{q+1}{2}\right) \alpha\right\}, \\
\boldsymbol{B} & =\frac{\lambda}{q+1}\left\{1+\frac{1-l_{\mathrm{z}}^{2}}{\omega_{\mathrm{cc}}^{2}}\right\}
\end{aligned}
$$


The above results can be compared with the results in Pakzad (2011a, c) in unmagnetized plasmas for planar and nonplanar geometry, respectively. Our results are also in agreement with the outcomes for the propagation of electron-acoustic waves in the presence of trapped electrons as reported in Mamun et al. (2002).

In order to study a stationary solitary wave solution for Eq. (13), we assume that the stationary solution can be expressed as $\varphi_{1}=\varphi_{1}(\chi)$, where $\chi=\xi-u \tau$. Substituting this expression into Eq. (13), the following stationary solitary wave solution can be obtained:

$\varphi_{1}=\varphi_{0} \sec h^{2}\left(\frac{\chi}{w}\right)$,

where $\varphi_{0}=3 u / A$ is the soliton amplitude and $w=2 \sqrt{\boldsymbol{B} / u}$ is its width.

The electron-acoustic soliton in Eq. (15), which is obtained in the small amplitude approximation, clearly indicates the existence of solitary waves with negative amplitude. $A$ is positive if $q>\frac{3(1+\alpha)}{1-3 \alpha}$. Figure 1 shows the region in which $A$ is positive. This figure shows that both positive and negative solitary waves can be created in this media. However for a small set of the medium parameters, positive amplitude solitons can be propagated (which has been marked in Fig. 1).

The soliton maximum amplitude is independent of the magnitude of the external magnetic field as one can observe in other similar plasma systems (Mamun et al., 2002; Anowar and Mamun, 2008). It is obvious that the magnetic field cannot change the energy of a physical system. Equation (14) shows that the parameter $A$ is proportional to $l_{\mathrm{z}}\left(l_{\mathrm{z}}=\cos \gamma\right.$, where $\gamma$ is the angle between the directions of the wave propagation vector $\boldsymbol{k}$ and the external magnetic field $\boldsymbol{B}_{0}$ ). Therefore $\varphi_{0}$ is inversely proportional to $l_{\mathrm{z}}$. It is interesting to know that the soliton maximum amplitude is inversely proportional to $l_{\mathrm{z}}^{2}$ in plasmas containing electrons with nonisothermal distribution (Mamun et al., 2002). On the other hand, Eq. (14) indicates that the soliton amplitude is also inversely proportional to $\alpha . \varphi_{0}$ is a complicated function of $q$, and one can find the effect of this parameter on the soliton amplitude using numerical analysis.

For $1-l_{\mathrm{z}}^{2} \ll \omega_{\mathrm{cc}}^{2}$ the soliton width is almost independent of $\omega_{\mathrm{cc}}$ and directly proportional to $\sqrt{l_{\mathrm{z}}}$. For $1-l_{\mathrm{z}}^{2} \gg \omega_{\mathrm{cc}}^{2}$ the soliton width is very sensitive to $\omega_{\mathrm{cc}}$. These results are in agreement with the results for nonisothermal distributions (Mamun et al., 2002).

\section{Results and discussions}

Numerical analysis has been employed to study the effects of nonextensive hot electrons, the density ratio between hot and cold electrons $(\alpha)$ and external magnetic field on the characteristics of solitary waves.

Figure 2 shows how the amplitude $\varphi_{0}$ changes with respect to the propagation angle, for different values of nonex-

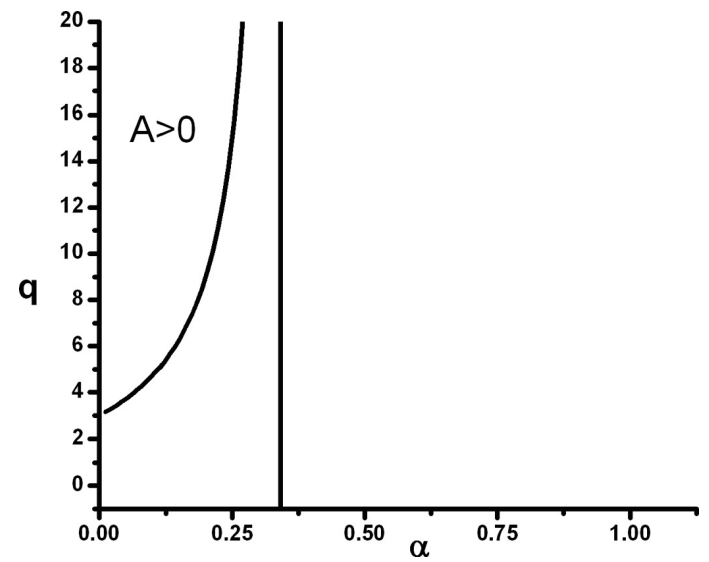

Fig. 1. Parameter $A$ in $q-\alpha$ space.

tensive parameter. Absolute value of the soliton amplitude $\varphi_{0}$ increases when $q$ increases (i.e. the long-range interaction between hot electrons is not negligible). Increasing the soliton amplitude is more sensible for the larger values of $\gamma$ (smaller values of $l_{\mathrm{z}}$ ). This figure also shows that the soliton amplitude is an increasing function of $\gamma$. Thus the soliton amplitude finds its maximum values in smaller values of $l_{\mathrm{Z}}$ as mentioned before.

The general shape of the $\varphi_{0}$ as a function of $\gamma$ but with greater values of the $q$ is the same as what we can see in Fig. 2. The difference is that the increasing ratio in the absolute value of soliton amplitude becomes smaller when $q$ increases (i.e. nonextensivity properties are negligible.). Note that nonextensive distribution goes marginally toward the Maxwellian distribution in greater values of $q$. Therefore one can conclude that the existence of nonextensive distributed hot electrons decreases the soliton amplitude. Also $\varphi_{0}$ is a sensitive function of the population of hot electrons when the concentration of nonextensive electrons becomes higher. The soliton energy is proportional to its amplitude. Thus we can conclude that $q$-distributed hot electrons decrease the soliton energy.

Figure $3 \mathrm{a}$ and $\mathrm{b}$ present maximum soliton amplitude $\varphi_{0}$ as a function of $\gamma$ with different values of $\alpha$. Figure 3a has been plotted with a negative $q$ value $(q=-0.5)$, while Fig. $3 \mathrm{~b}$ is the same as Fig. 3a but with a positive $q(q=0.5)$. These figures clearly show that the absolute value of the soliton peak decreases with an increasing $\alpha$. Comparison of Fig. 3a and $\mathrm{b}$ shows that the decreasing rate in the soliton amplitude is more sensible for greater values of $q$. Therefore sensibility to the ratio of hot to cold electrons decreases in a plasma system with $q$-distributed hot electrons. On the other hand, the soliton energy decreases when the population of hot electrons (relative to the cold electrons) increases, as Fig. 3a and $\mathrm{b}$ demonstrate.

Variation of the soliton width with respect to $\gamma$ has been plotted in Fig. 4 for different values of $q$. In general, the soliton width has a maximum value in the variate range of $\gamma$. This figure shows that the maximum value of the soliton 


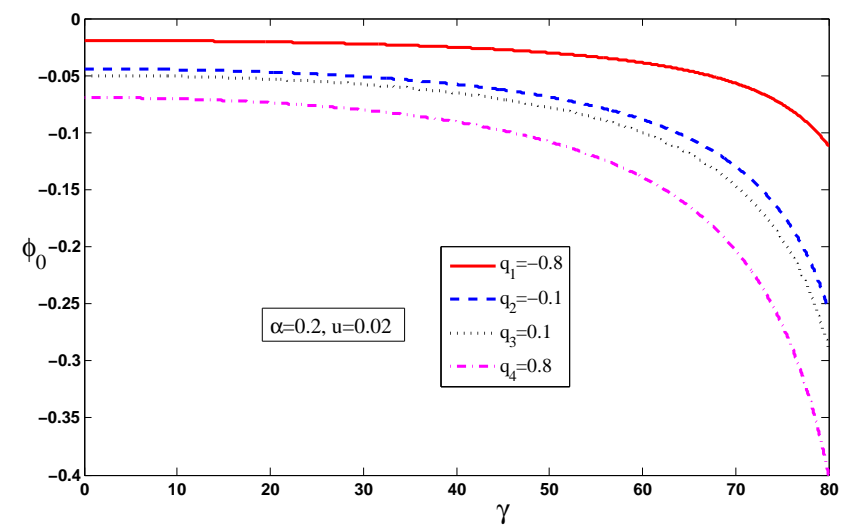

Fig. 2. Soliton amplitude $\varphi_{0}$ with respect to $\gamma$ with different values for the parameter $q$.

(a)
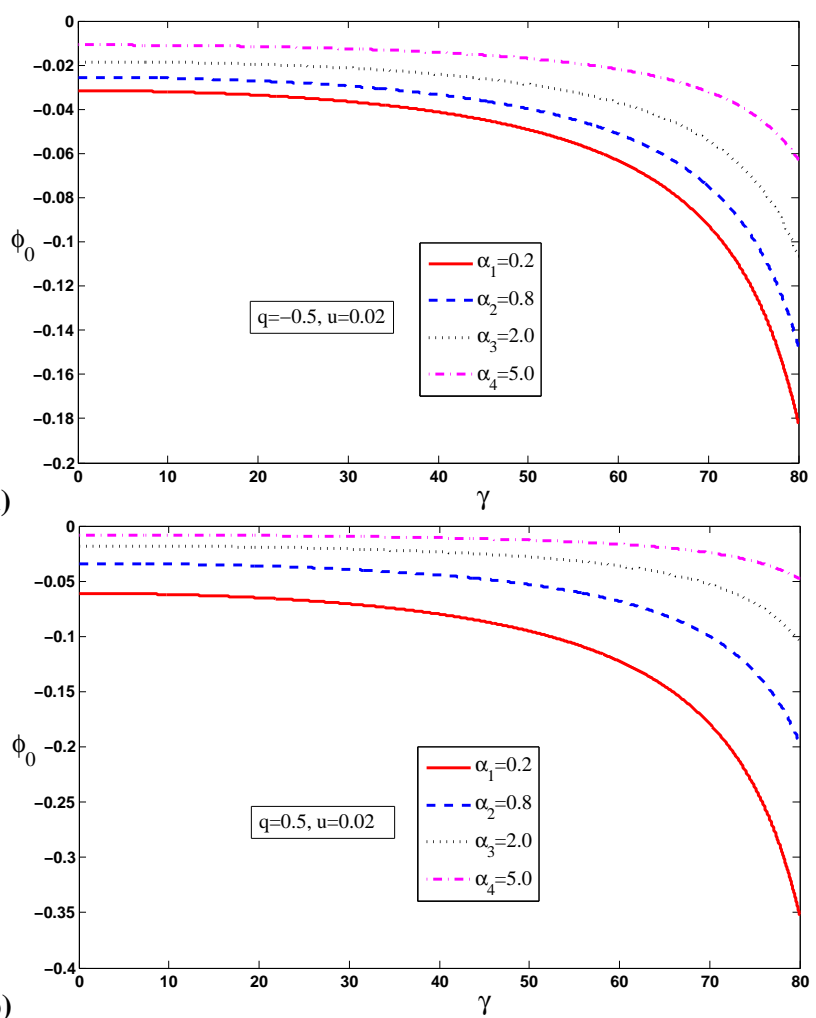

Fig. 3. (a) Maximum amplitude $\varphi_{0}$ with respect to $\gamma$ with different values for the parameter $\alpha$. (b) Maximum amplitude $\varphi_{0}$ with respect to $\gamma$ with different values for the parameter $\alpha$.

width decreases as $q$ increases. It is mentioned that nonextensive distribution reduces to Maxwellian distribution for large values of $q$. This means that the effect of nonextensivity is noticeable for smaller values of $q$. Therefore, one can conclude that the presence of nonextensive electrons increases the width of the soliton. It is obvious that rapid electrons have greater mean free path, thus decreasing the soliton compactness. Also one can find from Fig. 4 that the variation of $q$ value does not change the angle in which the maximum soliton width appears.

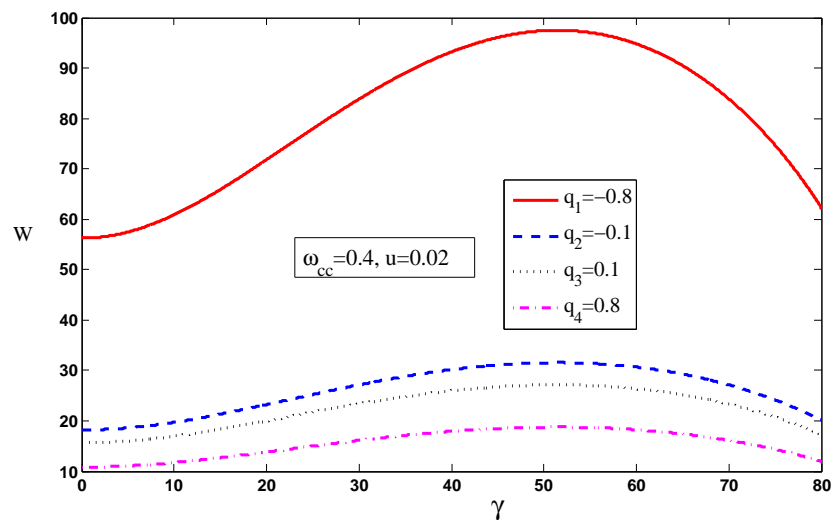

Fig. 4. Soliton width as functions of $\gamma$ with different values for the parameter $q$.

(a)

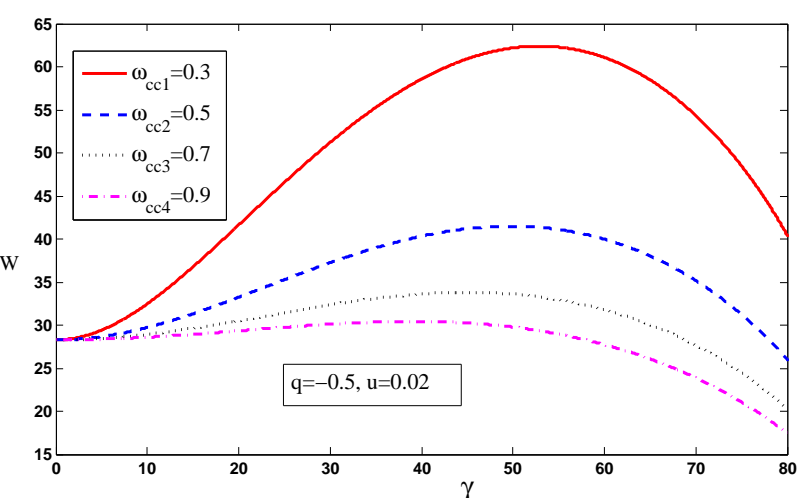

(b)

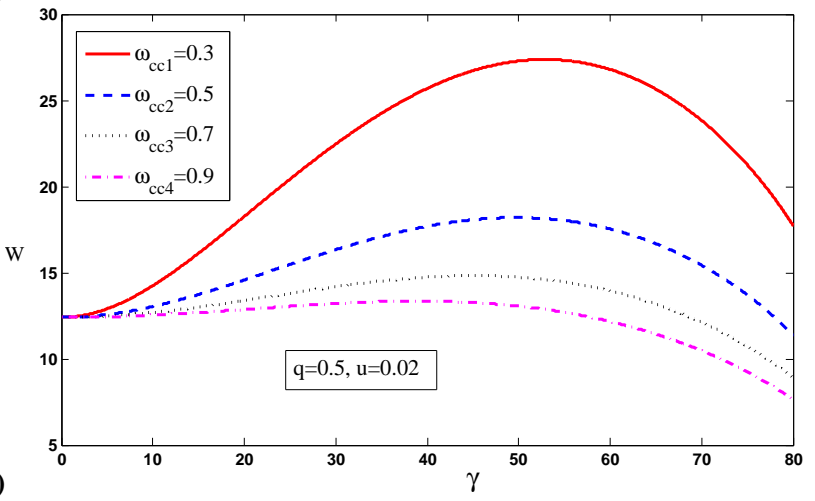

Fig. 5. (a) Soliton width with respect to $l_{\mathrm{Z}}$ with $q=-0.5$ and different values for the parameter $\omega_{\mathrm{cc}}$. (b) Soliton width with respect to $\gamma$ with $q=0.5$ and different values for the parameter $\omega_{\mathrm{cc}}$.

Figure $5 \mathrm{a}$ and $\mathrm{b}$ demonstrate the width of the soliton with respect to $\gamma$ with different values of the parameter $\omega_{\mathrm{cc}}$. Figure $5 \mathrm{a}$ has been plotted with $q=-0.5$, while Fig. $5 \mathrm{~b}$ has been sketched with $q=0.5$. This figure demonstrates that the soliton width decreases with an increasing $\omega_{\mathrm{cc}}$. Therefore a soliton finds smaller width in greater magnetic field. It is not surprising, because greater magnetic field reduces the radius of particle circular motion. It is obvious that for $\gamma=0$ the soliton width is not depending on the $\omega_{\mathrm{cc}}$ as also presented in Fig. 5a and b. 


\section{Conclusions}

Properties of electron-acoustic solitary waves propagating in magnetized plasmas of cold fluid ions and electrons with nonextensive distribution have been investigated in this paper. It may be noted that Landau damping of electrostatic plasma waves is often enhanced in the presence of a $q$-distributed electron population, as compared with Maxwellian plasmas. It is found that both rarefactive (negative amplitude) and compressive (positive amplitude) solitons can be propagated in this plasma. The soliton amplitude increases with an increasing value of the parameter $q$. This means that the long-range interaction effects reduce the soliton amplitude. It is the same as what we can see for superthermal, distributed electrons. It is obvious because the superthermal distribution is a special case of $q$-nonextensive distribution indeed. Soliton amplitude also increases with increasing values for angle $\gamma$. This means that the soliton energy increases in the presence of $q$-distributed electrons and also greater values of the angle $\gamma$. Solitons in plasmas with greater values of $q$ have steeper profiles. On the other hand solitons in plasmas with greater values of $\alpha$ have smaller maximum amplitude and therefore smaller energy. Also it is shown that the soliton profile becomes narrower in stronger magnetic fields.

As mentioned before both rarefactive and compressive solitons may be created in the plasma under study. This means that the parameter $A$ takes both positive and negative values. Thus it is possible that $A$ becomes zero. In this situation the KdV equation is not valid. It is an interesting and important point which can be investigated in further investigations.

Edited by: V. Carbone

Reviewed by: three anonymous referees

\section{References}

Amour, R. and Tribeche, M.: Variable charge dust acoustic solitary waves in a dusty plasma with a q-nonextensive electron velocity distribution, Phys. Plasmas, 7 pp., doi:10.1063/1.3428538, 2010.

Anowar, M. G. M. and Mamun, A. A.: Multidimensional instability of electron-acoustic solitary waves in a magnetized plasma with vortexlike electron distribution, Phys. Plasmas, 15, 6 pp., doi:10.1063/1.3006087, 2008.

Armstrong, T. P., Paonessa, M. T., Bell, E. V., and Krimigis, S. M.: Voyager observations of Saturnian ion and electron phase space densities, J. Geophys. Res., 88, 12 pp., doi:10.1029/JA088iA11p08893, 1983.

Bale, S. D., Kellogg, P. J., Larson, D. E., Lin, R. P., Goetz, K., and Lepping, R. P.: Bipolar electrostatic structures in the shock transition region: Evidence of electron phase space holes, Geophys. Res. Lett., 25, 4 pp., doi:10.1029/98GL02111, 1998.

Berthomier, M., Pottelette, R., Malingre, M., and Khotyainsev, Y.: Electron-acoustic solitons in an electron-beam plasma system,
Phys. Plasmas, 7, 8 pp., doi:10.1063/1.874150, 2000.

Cattaert, T., Verheest, F., and Hellberg, M. A.: Relativistic breakdown in planetary atmospheres, Phys. Plasmas, 12, 17 pp. doi:10.1063/1.2709652, 2005.

Cattell, C. A., Dombeck, J., Wygantet, J. R., Hudson, M. K., Mozer, F. S., Temerin, M. A., Peterson, W. K., Kletzing, C. A., Russell, C. T., and Pfaff, R. F.: Comparisons of Polar satellite observations of solitary wave velocities in the plasma sheet boundary and the high altitude cusp to those in the auroral zone, Geophys Res. Lett., 26, 4 pp., doi:10.1029/1998GL900304, 1999.

Chame, A. and Mello, E. V. L.: The Onsager reciprocity relations within Tsallis statistics, Phys. Lett. A, 228, 5 pp., doi:10.1016/S0375-9601(97)00125-4, 1997.

Danehkar, A., Saini, N. S., Hellberg, M. A., and Kourakis, I.: Electron-acoustic solitary waves in the presence of a suprathermal electron component, Phys. Plasmas, 18, 10 pp., doi:10.1063/1.3606365, 2011.

Derfler, H. and Simonen, T. C.: Higher-Order Landau Modes, Phys. Fluids, 12, 269-278, doi:10.1063/1.1692477, 1969.

Dubouloz, N., Pottelette, R., Malingre, M., and Treumann, R. A.: Turbulence generated by a gas of electron acoustic solitons, Geophys. Res. Lett., 18, 8 pp., doi:10.1029/93JA01611, 1991.

Dubouloz, N., Treumann, R. A., Pottelette, R., and Malingre, M.: Turbulence generated by a gas of electron acoustic solitons, J. Geophys. Res., 98, 8 pp., doi:10.1029/93JA01611, 1993.

Feldman, W. C., Anderson, R. C., Bame, S. J., Gary, S. P., Gosling, J. T., McComas, D. J., Thomsen, M. F., Paschmann, G., and Hoppe, M. M.: Electron velocity distributions near the Earth's bow shock, J. Geophys. Res., 88, 15 pp., doi:10.1029/JA088iA01p00096, 1983.

Fried, B. D. and Gould, R. W.: Longitudinal Ion Oscillations in a Hot Plasma, Phys. Fluids, 4, 9 pp., doi:10.1063/1.1706174, 1961.

Gary, S. P. and Tokar, R. L.: The electron-acoustic mode, Phys. Fluids, 28, 3 pp., doi:10.1063/1.865250, 1985.

Gill, T. S., Kaur, H., and Saini, N. S.: Small amplitude electron-acoustic solitary waves in a plasma with nonthermal electrons, Chaos Soliton. Fract., 30, 5 pp., doi:10.1016/j.chaos.2005.09.070, 2006.

Gougam, L. A. and Tribeche, M.: Weak ion-acoustic double layers in a plasma with a $q$-nonextensive electron velocity distribution, Astrophys. Space Sci., 331, 9 pp., doi:10.1007/s10509-0100447-2, 2011.

Henry, D. and Treguier, J. P.: Propagation of electronic longitudinal modes in a non-Maxwellian plasma, J. Plasma Phys., 8, 9 pp., doi:10.1017/S0022377800007169, 1972.

Ikezawa, S. and Nakamura, Y.: Observation of Electron Plasma Waves in Plasma of Two-Temperature Electrons, J. Phys. Soc. Jpn., 50, 6 pp., doi:10.1143/JPSJ.50.962, 1981.

Kakad, A. P., Singh, S. V., Reddy, R. V., Lakhina, G. S., and Tagare, S. G.: Electron acoustic solitary waves in the Earth's magnetotail region, Adv. Space Res., 43, 5 pp., doi:10.1016/j.asr.2009.03.005, 2009.

Kaniadakis, G., Lavagno, A., and Quarati, P.: Generalized Statistics and Solar Neutrinos, Phys. Lett. B, 369, 5 pp., doi:10.1016/03702693(95)01535-3, 1996.

Lakhina, G. S., Kakad, A. P., Singh, S. V., and Verheest, F.: Ionand electron-acoustic solitons in two-electron temperature space plasmas, Phys. Plasmas, 15, 7 pp., doi:10.1063/1.2930469, 2008. 
Lakhina, G. S., Singh, S. V., Kakad, A. P., Goldstein, M. L., Viñas, A. F., and Pickett, J. S.: A mechanism for electrostatic solitary structures in the Earth's magnetosheath, J. Geophys. Res., 114, 5 pp., doi:10.1029/2009JA014306, 2009.

Lavagno, A., Kaniadakis, G., Rego-Monteiro, M., Quarati, P., and Tsallis, C.: Non-extensive thermostatistical approach of the peculiar velocity function of galaxy clusters, Astrophys. Lett. Comm., 35, 449-455, 1998.

Lenzi, E. K., Malacarne, L. C., and Mendes, R. S.: Perturbation and Variational Methods in Nonextensive Tsallis Statistics, Phys. Rev. Lett., 80, 4 pp., doi:10.1103/PhysRevLett.80.218, 1998.

Leubner, M. P.: On Jupiter's whistler emission, J. Geophys. Res., 87, 6335-6338, doi:10.1029/JA087iA08p06335, 1982.

Leubner, M. P. and Schupfer, N.: Mirror instability thresholds in suprathermal space plasmas, J. Geophys. Res., 105, 5 pp., doi:10.1029/1999JA000447, 2000.

Lima, J. A. S., Silva, R., and Santos, J.: Plasma oscillations and nonextensive statistics, Phys. Rev. E, 61, 4 pp., doi:10.1103/PhysRevE.61.3260, 2000.

Lin, C. S., Burch, J. L., Shawhan, S. D. and Gurnett, D. A.:Correlation of auroral hiss and upward electron beams near the polar cusp, J. Geophys. Res., 89, 11 pp., doi: 10.1029/JA089iA02p00925, 1984.

Mace, R. L. and Hellberg, M. A.: The Korteweg-de VriesZakharov-Kuznetsov equation for electron-acoustic waves, Phys. Plasmas, 8, 8 pp., doi:10.1063/1.1363665, 2001.

Mace, R. L., Baboolal, S., Bharuthram, R., and Hellberg, M. A.: Arbitrary-amplitude electron-acoustic solitons in a twoelectron-component plasma, J. Plasma Phys., 45, 6 pp., doi:10.1017/S0022377800015749, 1991.

Mamun, A. A. and Shukla, P. K.: Electron-acoustic solitary waves via vortex electron distribution, J. Geophys. Res., 107, 5 pp., doi:10.1029/2001JA009131, 2002.

Mamun, A. A., Shukla, P. K., and Stenflo, L.: Obliquely propagating electron-acoustic solitary waves, Phys. Plasmas, 9, 4 pp., doi:10.1063/1.1462635, 2002.

Matsumoto, H., Kojima, H., Miyatake, T., Omura, Y., Okada, M., Nagano, I., and Tsutsui, M.: Electrostatic solitary waves (ESW) in the magnetotail: BEN wave forms observed by GEOTAIL, Geophys. Res. Lett., 21, 4 pp., doi:10.1029/94GL01284, 1994.

Mendis, D. A. and Rosenberg, M.: Cosmic Dusty Plasma, Annu. Rev. Astron. Astr., 32, 45 pp., doi:10.1146/annurev.aa.32.090194.002223, 1994.

Pakzad, H. R.: Effect of q-nonextensive distribution of electrons on electron acoustic solitons, Astrophys. Space Sci., 333, 9 pp., doi:10.1007/s10509-010-0570-0, 2011a.

Pakzad, H. R.: Effect of q-nonextensive electrons on electron acoustic solitons, Phys. Scr., 83, 4 pp., doi:10.1088/00318949/83/01/015505, 2011b.

Pakzad, H. R.: Nonplanar ion-acoustic solitary waves with superthermal electrons in warm plasma, Phys. Plasmas, 18, 5 pp., doi:10.1063/1.3622207, 2011c.

Pakzad, H. R. and Tribeche, M.: Electron-acoustic solitons in plasma with nonthermal electrons, Astrophys. Space Sci., 330, 5 pp., doi:10.1007/s10509-010-0367-1, 2010.

Pakzad, H. R. and Tribeche, M.: Electron acoustic double layers in a plasma with a q-nonextensive electron velocity distribution, Astrophys Space Sci., 330, 95-99, doi:10.1007/s10509-010-0367$1,2011$.
Plastino, A. R. and Plastino, A.: Stellar polytropes and Tsallis' entropy, Phys. Lett. A, 174, 3 pp., doi:10.1016/03759601(93)90195-6, 1993.

Plastino, A. R., Plastino, A., and Tsallis, C.: The classical N-body problem within a generalized statistical mechanics, J. Phys. AMath. Gen., 27, 9 pp., doi:10.1088/0305-4470/27/17/008, 1994.

Pottelette, R. and Berthomier, M.: Nonlinear electron acoustic structures generated on the high-potential side of a double layer, Nonlin. Processes Geophys., 16, 373-380, doi:10.5194/npg-16-3732009, 2009.

Rajagopal, A. K.: The Sobolev inequality and the Tsallis entropic uncertainty relation, Phys. Lett. A., 205, 5 pp., doi:10.1016/0375-9601(95)00500-3, 1995.

Rajagopal, A. K.: Dynamic Linear Response Theory for a Nonextensive System Based on the Tsallis Prescription, Phys. Rev. Lett., 76, 5 pp., doi:10.1103/PhysRevLett.76.3469, 1996.

Renyi, A.: On a new axiomatic theory of probability, Acta Math. Hung., 6, 41 pp., doi:10.1007/BF02024393, 1955.

Singh, S. V. and Lakhina, G. S.:Generation of electron-acoustic waves in the magnetosphere, Planet. Space Sci., 49, 8 pp., doi:10.1016/S0032-0633(00)00126-4, 2001.

Tagare, S. G., Singh, S. V., Reddy, R. V., and Lakhina, G. S.: Electron acoustic solitons in the Earth's magnetotail, Nonlin. Processes Geophys., 11, 215-218, doi:10.5194/npg-11-215-2004, 2004.

Thomsen, M. F., Barr, H. C., Gary, S. P., Feldman, W. C., and Cole, T. E.: Stability of electron distributions within the Earth's bow shock, J. Geophys. Res., 88, 11 pp., doi:10.1029/JA088iA04p03035, 1983.

Tokar, R. L. and Gary, S. P.: Electrostatic hiss and the beam driven electron acoustic instability in the dayside polar cusp, Geophys. Res. Lett., 11, 4 pp., doi: 10.1029/GL011i012p01180, 1984.

Torres, D. F., Vucetich, H., and Plastino, A.: Early Universe Test of Nonextensive Statistics, Phys. Rev. Lett., 79, 3 pp., doi:10.1103/PhysRevLett.79.1588, 1997.

Tsallis, C.: Possible generalization of Boltzmann-Gibbs statistics, J. Stat. Phys., 52, 9 pp., doi:10.1007/BF01016429, 1988.

Vasyliunas, V. M.: A survey of low-energy electrons in the evening sector of the magnetosphere with OGO 1 and OGO 3, J. Geophys. Res., 73, 2839-2884, doi:10.1029/JA073i009p02839, 1968.

Verheest, F., Cattaert, T., and Hellberg, M. A.: Compressive and rarefactive electron-acoustic solitons and double layers in space plasmas, Space Sci. Rev., 121, 13 pp., 2005.

Verheest, F., Hellberg, M. A., and Lakhina, G. S.: Necessary conditions for the generation of acoustic solitons in magnetospheric and space plasmas with hot ions, Astrophys. Space Sci. Trans., 3, 15-20, doi:10.5194/astra-3-15-2007, 2007.

Watanabe, W.: Ion-acoustic solitons excited by a single grip, J. Plasma Phys., 14, 12 pp., doi:10.1017/S0022377800009648, 1975.

Watanabe, K. and Taniuti, T.: Electron-Acoustic Mode in a Plasma of Two-Temperature Electrons, J. Phys Soc. Jpn., 43, 1819-1820, doi:10.1143/JPSJ.43.1819, 1977.

Yu, M. Y. and Shukla, P. K.: Linear and nonlinear modified electron-acoustic waves, J. Plasma Phys., 29, 5 pp., doi:http://dx.doi.org/10.1017/S0022377800000866, 1983. 